information centres. Nevertheless, the onset of recovery was rapid. The important late effect of the poisoning was the development of jejunal ulceration and diverticula formation, and hence the formation of strictures remains a real possibility. The induction of a diuresis seemed to play a particularly important part in preventing acute renal damage.

The prognosis in methylene chloride poisoning then is far from hopeless. With scrupulous intensive care recovery may be anticipated.

We thank Dr H M Leather for permission to report on a patient under his care.

${ }^{1}$ Hughes, J P, fournal of the American Medical Association, 1954, 156, 234

2 Stewart, R D, American fournal of Nursing, 1967, 67, 85

${ }^{3}$ Dykes, M H M, International Anesthesiology Clinics, 1970, 357.

Freedom Fields Hospital, Plymouth

C J C ROBERTS, MB, MRCP, medical registrar

F P F MARSHALL, MB, BCHIR, senior house officer

\section{Rosacea and migraine}

An association between certain clinical conditions may remain unrecognised for years. There are many ways with which the skin and other systems are linked. Examination of the skin may help the clinician in assessing systemic disease: many patients with dermatitis herpetiformis have intestinal malabsorption and patients with vitiligo show an increase of organ-specific autoimmune diseases. ${ }^{1}$ During our investigation into a new approach to the treatment of facial flushing in rosacea we found three consecutive patients who not only had rosacea but also migraine. This stimulated us to attempt to establish the association between the two conditions.

\section{Methods and results}

One hundred and thirty-seven patients with rosacea and 161 controls were asked whether they had migraine. The control group were predominantly fit hospital personnel; a few had either viral warts, basal cell carcinoma, or gravitational eczema. The definition of migraine was episodic vascular headache with at least two of the following features: unilateral headache; associated nausea or vomiting; visual aura; family history of migraine; and past history of bilious attacks. ${ }^{2}$ The patients with rosacea were mainly women, 89 women and 48 men, aged $20-87$ (mean $46.0 \pm 1.3$ years). The controls were not significantly different: 114 women and 47 men, aged $18-80$ (mean $42 \cdot 0 \pm 3 \cdot 1$ years).

Sixty of the 137 patients with rosacea $(44 \%)$ had suffered from migraine; this contrasted with 21 of the 161 controls $(13.1 \%)$. There was thus a significant association $(P<0.0005)$ between migraine and rosacea (see table). Twenty-nine of the controls had intermittent facial flushing with no other features of rosacea and 16 of these had migraine. Exclusion of these controls further increased the significance of the association between rosacea and migraine.

Incidence of migraine

\begin{tabular}{|c|c|c|c|}
\hline & Rosacea & Controls & Significance \\
\hline $\begin{array}{l}\text { With migraine } \\
\text { No migraine }\end{array}$ & $60\left(44{ }^{\circ}\right)$ & $21\left(13^{\prime \prime}\right)$ & $P<0.0005$ \\
\hline Total & 137 & 161 & \\
\hline
\end{tabular}

\section{Discussion}

We know of no report in which the association between migraine and rosacea has been described. Crawford ${ }^{3}$ reported a 29-year-old woman with migraine who had an unusual eruption and flushing of the right leg, occurring independently of headaches, possibly representing a cutaneous equivalent. Facial migraine-which consists of episodic facial neuralgia, flushing, and reddening of the skin and lachrymation-usually occurs in middle-aged women with a past history of migraine ${ }^{4}$ the presence or absence of rosacea has not been reported in these patients.

The incidence of migraine in our controls is higher than that in other control series. ${ }^{5}$ Thus our observation that $44 \%$ of patients with rosacea suffered from migraine is significant when compared with not only our own but also other control series. The association between rosacea and migraine is not surprising, since both are associated with abnormal vascular reactivity, and both are aggravated by stress. Although migraine tends to occur in younger patients than rosacea, this lack of a temporal relationship does not make the association less likely because it is also seen in other conditions such as eczema and asthma.

The association between rosacea and migraine has several implications. The presence of rosacea may help clinicians in diagnosing migraine variants. Oral contraceptives may significantly increase the frequency and severity of headaches in migrainous women, and hence should patients with rosacea avoid them ? Some patients with migraine develop their attacks after eating cheese or chocolate because of failure to metabolise such compounds as tyramine or phenylethylamine. Do such factors aggravate rosaceous flushing? Study of the facial blood vessels in rosacea may help to understand better the physiological and biochemical abnormalities of the intracranial, extracerebra blood vessels of migraine. Conversely, could drugs prescribed for migraine help facial flushing in rosacea? In low doses clonidine reduces facial flushing and helps migraine at the menopause, and our preliminary investigations show that clonidine probably helps in reducing the facial erythema and flushing of rosacea.

We thank Dr N R Rowell and Dr J A Cotterill for access to patients and Mrs L Lane, Miss S Sharp, and Mrs J Hodson for secretarial help.

${ }^{1}$ Cunliffe, W J, et al, British fournal of Dermatology, 1968, 80, 135.

2 World Federation cf Neurology Research Group on Migraine and Headache in Background to Migraine, p 181. London, Heinemann, 1969.

${ }^{3}$ Crawford, P F, British fournal of Dermatology, 1961, 73, 419.

4 McArdle, J, in Background to Migraine. London, Heinemann, 1969.

${ }^{5}$ Brewis, M, et al, Acta Neurologica Scandinavica, 1966. 42, Suppl 24.

Departments of Dermatology, The General Infirmary, Leeds and $S$ James's (University) Hospital, Leeds

TAN, S G, MB, MRCP, registrar in dermatology

CUNLIFFE, W J, MD, MRCP, consultant dermatologist

\section{Intestinal obstruction complicating orphenadrine treatment}

Gastrointestinal symptoms associated with drugs are common in clinical practice and may take several forms. A reduction in bowel motility with consequent constipation may be caused by drugs with an atropine-like action, including anti-Parkinsonian agents. Parkinsonism itself may cause constipation, ${ }^{1}$ and treatment with a constipation-provoking agent might be expected to produce troublesome symptoms. Severe constipation is perhaps less common than would be predicted but can occasionally be severe enough to cause intestinal obstruction. ${ }^{2}$ A case is presented here in which orphenadrine appears to have precipitated such an event, an association not previously reported.

\section{Case report}

The patient was a 77-year-old man who was diagnosed as suffering from Parkinsonism in 1972, when immobile facies, bradykinesia, and a typical tremor were noted. Orphenadrine was started at a dose of $50 \mathrm{mg}$ three times a day, which improved his symptoms. In 1973 an episode of urinary retention was treated by transurethral resection of the prostate, with complete 